\title{
Construction of a quantum field theory in four dimensions
}

\author{
Harald Grosse \\ Fakultät für Physik, Universität Wien, Boltzmanngasse 5, A-1090 Wien, Austria \\ E-mail: harald.grosse@univie.ac.at

\section{Raimar Wulkenhaar*} \\ Mathematisches Institut der WWU, Einsteinstr. 62, D-48149 Münster, Germany \\ E-mail: raimar@math.uni-muenster.de
}

\begin{abstract}
We summarise our recent construction of the $\lambda \phi_{4}^{4}$-model on four-dimensional Moyal space. In the limit of infinite noncommutativity, this model is exactly solvable in terms of the solution of a non-linear integral equation. Surprisingly, this limit describes Schwinger functions of a Euclidean quantum field theory on standard $\mathbb{R}^{4}$ which satisfy the easy Osterwalder-Schrader axioms boundedness, invariance and symmetry. The decisive reflection positivity axiom is, for the 2-point function, equivalent to the question whether the solution of the integral equation is a Stieltjes function. A numerical investigation confirms this for coupling constants $\lambda_{c}<\lambda \leq 0$ with $\lambda_{c} \approx-0.39$.
\end{abstract}

Frontiers of Fundamental Physics 14 - FFP14,

15-18 July 2014

Aix Marseille University (AMU) Saint-Charles Campus, Marseille

\footnotetext{
*Speaker.
} 


\section{Introduction}

The construction of a 4D quantum field theory [1] is a major open problem of mathematical physics. In this note we review a sequence of papers $[2,3,4]$ in which we successfully used symmetry and fixed point methods to exactly solve a toy model for a 4D QFT.

We follow the Euclidean approach, starting from a partition function with source term $\mathscr{Z}[J]$. This involves the action functional of the model, but regularised in finite volume $V$ and with finite energy cut-off $\Lambda$. Mostly, these regularisations destroy the symmetries of the model and have to be restored in the end. Our toy model is characterised by a huge symmetry group even in presence of regularisation. The resulting constraints lead to a complete solution of the model.

We start from the usual $\lambda \phi_{4}^{4}$-model with action $\int_{\mathbb{R}^{4}} d x\left(\frac{1}{2} \phi\left(-\Delta+\mu^{2}\right) \phi+\frac{\lambda}{4} \phi^{4}\right)(x)$. Finite volume is achieved through a harmonic oscillator potential. The energy cut-off $\Lambda$, or a minimal length scale $\frac{1}{\Lambda}$, typically makes the model non-local. A convenient choice is to replace the pointwise product by the Moyal product $(f \star g)(x)=\int_{\mathbb{R}^{4} \times \mathbb{R}^{4}} \frac{d k d y}{(2 \pi)^{4}} f\left(x+\frac{1}{2} \Theta k\right) g(x+y) e^{\mathrm{i} k y}$, where $\Theta$ is a skewsymmetric $4 \times 4$-matrix. Schwartz class functions with Moyal product can be mapped to infinite matrices with rapidly decaying entries, and the energy cutoff $\Lambda$ consists in a finite size $\mathscr{N}$ of these matrices. The regulated action thus reads

$$
S[\phi]=\frac{1}{64 \pi^{2}} \int d^{4} x\left(\frac{Z}{2} \phi \star\left(-\Delta+\Omega^{2}\left\|2 \Theta^{-1} x\right\|^{2}+\mu_{\text {bare }}^{2}\right) \phi+\frac{\lambda_{\text {bare }} Z^{2}}{4} \phi \star \phi \star \phi \star \phi\right)(x),
$$

where $Z, \lambda_{\text {bare }}, \mu_{\text {bare }}$ are functions of renormalised values $\lambda, \mu$ and of the regulators $\Omega, \Theta, \mathscr{N}$ encoded in the oscillator potential and the $\star$-product. Several limits can be discussed:

- $\Omega, \Theta, \frac{1}{N} \rightarrow 0$ : This is the pertubatively renormalisable, but trivial, $\lambda \phi_{4}^{4}$-model.

- $\Theta \neq 0$ fixed; $\Omega=0$ : This is often called "noncommutative $\lambda \phi_{4}^{4}$-theory", which is not renormalisable due to the UV/IR-mixing problem.

- $\Theta, \Omega_{\text {ren }} \neq 0$ fixed: A perturbatively renormalisable model [5] with ultraviolet fixed point $\Omega=1$ at which the $\beta$-function vanishes [6].

- $\Omega=1$ fixed; $\Theta, \mathscr{N} \rightarrow \infty$ : The limit studied here, giving rise to an exactly solvable model.

\section{Matrix model, Ward identity and Schwinger-Dyson equations}

At $\Omega=1$ the action (1.1) becomes self-dual under Langmann-Szabo transform and can be expressed as a quartic matrix model

$$
S[\phi]=V\left(\sum_{\underline{m}, \underline{n}, \underline{k} \in \mathbb{N}_{\mathcal{N}}^{2}} E_{\underline{m} \underline{\underline{n}}} \Phi_{\underline{\underline{n}} \underline{\underline{k}}} \Phi_{\underline{k} \underline{\underline{m}}}+\frac{Z^{2} \lambda}{4} \sum_{\underline{k}, \underline{l,}, \underline{\underline{m}, \underline{\mathbb{N}_{N}}}} \Phi_{\underline{\underline{k}} \underline{\underline{l}}} \Phi_{\underline{\underline{l}} \underline{\underline{m}}} \Phi_{\underline{m} \underline{\underline{n}}} \Phi_{\underline{\underline{n}} \underline{k}}\right),
$$

where $E_{\underline{m} \underline{\underline{n}}}=E_{|\underline{m}|} \delta_{\underline{m} \underline{n}}, E_{|\underline{m}|}:=Z\left(\frac{|\underline{m}|}{\sqrt{V}}+\frac{\mu_{\text {bare }}^{2}}{2}\right)$ and $V:=\left(\frac{\theta}{4}\right)^{2}$. Under $\mathbb{N}_{\mathscr{N}}^{2}$ we understand the set of pairs $\underline{m}=\left(m_{1}, m_{2}\right) \in \mathbb{N}^{2}$ with $|\underline{m}|:=m_{1}+m_{2} \leq \mathscr{N}$. The resulting partition function $\mathscr{Z}[J]=$ $\int \mathscr{D}[\Phi] \exp (-S[\Phi]+V \operatorname{tr}(\Phi J))$ is covariant under the unitary transformation $\Phi \mapsto U^{*} \Phi U$. This covariance gives rise to the following Ward identity [6]: 


$$
0=\sum_{\underline{n} \in \mathbb{N}_{\mathscr{N}}^{2}}\left(\frac{\left(E_{|\underline{a}|}-E_{|\underline{a}|}\right)}{V} \frac{\partial^{2} \mathscr{Z}}{\partial J_{\underline{a} \underline{n}} \partial J_{\underline{n} \underline{p}}}+J_{\underline{p} \underline{n}} \frac{\partial \mathscr{Z}}{\partial J_{\underline{a} \underline{n}}}-J_{\underline{n} \underline{a}} \frac{\partial \mathscr{Z}}{\partial J_{\underline{n} \underline{p}}}\right) .
$$

Perturbatively, Feynman graphs in matrix models are ribbon graphs which encode a genus- $g$ Riemann surface with $B$ boundary components. The $k^{\text {th }}$ boundary face is characterised by $N_{k} \geq 1$ external double lines to which we attach the source matrices $J$. Since $E$ is diagonal, the matrix index is conserved along each strand of the ribbon graph. Therefore, the right index of $J_{\underline{a} \underline{b}}$ coincides with the left index of another $J_{\underline{b} \underline{c}}$, or of the same $J_{\underline{b} \underline{b}}$. Accordingly, the $k^{\text {th }}$ boundary component carries a cycle $J_{P} \equiv J_{\underline{p}_{1} \ldots \underline{p}_{N_{k}}}^{N_{k}}:=\prod_{j=1}^{N_{k}} J_{\underline{p}_{j} \underline{p_{j+1}}}$ of $N_{k}$ external sources, with $N_{k}+1 \equiv 1$. Therefore, the logarithm of the partition function has the following expansion $\left(S_{N_{1} \ldots N_{B}}\right.$ is a symmetry factor):

$$
\log \frac{\mathscr{Z}[J]}{\mathscr{Z}[0]}=\sum_{B=1}^{\infty} \sum_{1 \leq N_{1} \leq \ldots \leq N_{B}}^{\infty} \sum_{\underline{p}_{1}^{\beta}, \ldots, \underline{p}_{N_{\beta}}^{\beta} \in I} \frac{V^{2-B}}{S_{N_{1} \ldots N_{B}}} G_{\left|\underline{p_{1}} \ldots \underline{p}_{N_{1}}^{1}\right| \ldots\left|\underline{p}_{1}^{B} \ldots \underline{p}_{N_{B}}^{B}\right|} \prod_{\beta=1}^{B}\left(\frac{1}{N_{\beta}} J_{\underline{p}_{1}^{\beta} \ldots \underline{p}_{N_{\beta}}^{\beta}}^{N_{\beta}}\right) .
$$

The cycle expansion (2.3) provides for external matrices $E$ of compact resolvent the kernel of multiplication by $E_{|\underline{a}|}-E_{|\underline{p}|}$ in (2.2):

Theorem 1 ([2])

$$
\begin{aligned}
& \sum_{\underline{n} \in \mathbb{N}_{\mathcal{N}}^{2}} \frac{\partial^{2} \mathscr{Z}[J]}{\partial J_{\underline{a n}} \partial J_{\underline{n} \underline{p}}}
\end{aligned}
$$

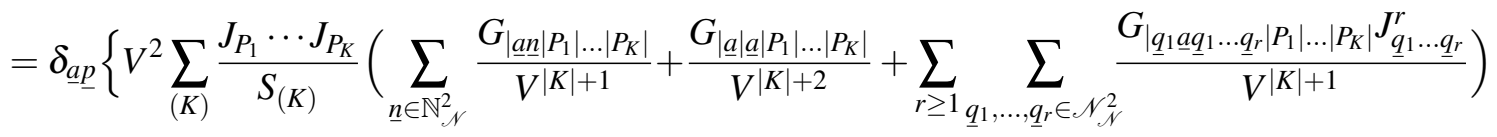

$$
\begin{aligned}
& \left.+V^{4} \sum_{(K),\left(K^{\prime}\right)} \frac{J_{P_{1}} \cdots J_{P_{K}} J_{Q_{1}} \cdots J_{Q_{K^{\prime}}}}{S_{(K)} S_{\left(K^{\prime}\right)}} \frac{G_{|\underline{a}| P_{1}|\ldots| P_{K} \mid}}{V^{|K|+1}} \frac{G_{|\underline{a}| Q_{1}|\ldots| Q_{K^{\prime}} \mid}}{V^{\left|K^{\prime}\right|+1}}\right\} \mathscr{Z}[J] \\
& +\frac{V}{E_{|\underline{p}|}-E_{|\underline{a}|}} \sum_{\underline{n} \in \mathbb{N}_{\mathscr{N}}^{2}}\left(J_{\underline{\underline{p}} \underline{\underline{p}}} \frac{\partial \mathscr{Z}[J]}{\partial J_{\underline{a} \underline{\underline{n}}}}-J_{\underline{n} \underline{a}} \frac{\partial \mathscr{Z}[J]}{\partial J_{\underline{\underline{n}} \underline{p}}}\right) \text {. }
\end{aligned}
$$

Formula (2.4) is the core of our approach. It is a consequence of the unitary group action and the cycle structure of the partition function. The possibility to kill two $J$-derivatives via (2.4) lets the usually infinite hierarchy of Schwinger-Dyson equations collapse [2]:

Proposition 2. In a scaling limit $V \rightarrow \infty$ with $\frac{1}{V} \sum_{\underline{n} \in \mathbb{N}_{N}^{2}}$ finite, the $(B=1)$-sector of $\log \mathscr{Z}$ reads

$$
\begin{aligned}
G_{|\underline{a} \underline{b}|} & =\frac{1}{E_{|\underline{a}|}+E_{\underline{|b|} \mid}}-\frac{\lambda}{E_{|\underline{a}|}+E_{|\underline{b}|}} \frac{1}{V} \sum_{\underline{p}_{\in \in \mathbb{N}^{2}}}\left(G_{|\underline{a} \underline{b}|} G_{|\underline{a} \underline{p}|}-\frac{G_{|\underline{p} \underline{b}|}-G_{|\underline{a} \underline{b}|}}{E_{|\underline{p}|}-E_{|\underline{a}|}}\right), \\
G_{\left|\underline{b}_{0} \underline{b}_{1} \ldots \underline{b}_{N-1}\right|} & =(-\lambda) \sum_{l=1}^{\frac{N-2}{2}} \frac{G_{\left|\underline{b}_{0} \underline{b}_{1} \ldots \underline{b}_{2 l-1}\right|} G_{\left|\underline{b}_{2} \underline{b}_{2 l+1} \ldots \underline{b}_{N-1}\right|}-G_{\left|\underline{b}_{2 l} \underline{b}_{1} \ldots \underline{b}_{2 l-1}\right|} G_{\left|\underline{b}_{0} \underline{b}_{2 l+1} \ldots \underline{b}_{N-1}\right|}}{\left(E_{\left|\underline{b}_{0}\right|}-E_{\left|\underline{b}_{2 l}\right|} \mid\left(E_{\left|\underline{b}_{1}\right|}-E_{\left|\underline{b}_{N-1}\right|}\right)\right.} .
\end{aligned}
$$

Equation (2.5) was first obtained in [7] by the graphical method proposed by [6]. The non-linearity of (2.5) was successfully addressed in [2,4]. The purely algebraic formula (2.6) for $N \geq 4$ relies, apart from (2.4), on the reality $\Phi=\Phi^{*}$ of the matrix model. Absence of index summations in (2.6) 
means that the $\beta$-function of the QFT defined by (1.1) vanishes identically, as proved perturbatively in [6]. The Schwinger-Dyson equations for functions $G_{\left|\underline{p}_{1}^{1} \ldots \underline{p}_{N_{1}}^{1}\right| \ldots\left|\underline{p}_{1}^{B} \ldots \underline{N}_{N_{B}}^{B}\right|}$ with $B>1$ are similar in the following sense: The basic functions with all $N_{i} \leq 2$ satisfy a complicated, but linear, equation. All higher functions with at least one $N_{i} \geq 3$ are purely algebraic.

\section{Renormalisation and integral representation}

The scaling limit $V \rightarrow \infty$ with $\frac{1}{V} \sum_{\underline{n} \in \mathbb{N}_{N}^{2}}$ finite turns discrete matrix indices into continuous variables and sums into integrals. These integrals diverge and therefore require an energy cutoff $a, b, \cdots \in\left[0, \Lambda^{2}\right]$. Normalisation conditions on the lowest Taylor terms of the two-point function $G_{|\underline{a b}|} \mapsto G_{a b}$ express the bare quantities $Z, \mu_{\text {bare }}$ in terms of renormalised values $\mathscr{Y}, \mu$ and of the cutoff $\Lambda^{2}$. Eliminating $Z, \mu_{\text {bare }}$ by their normalisation equations leads to a highly non-linear equation for the renormalised two-point function. The non-linearity cancels for the difference $G_{a b}-G_{a 0}$ if the finite wavefunction renormalisation is $1+\mathscr{Y}=-\left.\frac{d G_{0 b}}{d b}\right|_{b=0}$. These steps turn (2.5) into a linear singular integral equation of Carleman type. The solution theory of such equations gives:

Theorem 3 ([4]) The matrix 2-point function $G_{a b}$ of the $\lambda \phi_{4}^{\star 4}$-model is in infinite volume limit and for coupling constants $\lambda<0$ given in terms of the boundary 2-point function $G_{0 a}$ by

$$
G_{a b}=\frac{\sin \left(\tau_{b}(a)\right)}{|\lambda| \pi a} \exp \left(\operatorname{sign}(\lambda)\left(\mathscr{H}_{0}^{\Lambda}\left[\tau_{0}(\bullet)\right]-\mathscr{H}_{a}^{\Lambda}\left[\tau_{b}(\bullet)\right]\right)\right)
$$

where $\tau_{b}(a):=\underset{[0, \pi]}{\arctan }\left(\frac{|\lambda| \pi a}{b+\frac{1+\lambda \pi a \mathscr{H}_{a}^{\Lambda}\left[G_{0}\right]}{G_{0 a}}}\right)$ and $\mathscr{H}_{a}^{\Lambda}[f(\bullet)]:=\frac{1}{\pi} \lim _{\mathcal{E} \rightarrow 0}\left(\int_{0}^{a-\varepsilon}+\int_{a+\varepsilon}^{\Lambda^{2}}\right) \frac{f(q) d q}{q-a} d e-$ notes the finite Hilbert transform. The boundary function satisfies the fixed point equation

$$
G_{0 b}=\frac{1}{1+b} \exp \left(-\lambda \int_{0}^{b} d t \int_{0}^{\Lambda^{2}} \frac{d p}{(\lambda \pi p)^{2}+\left(t+\frac{1+\lambda \pi p \mathscr{H}_{p}^{\Lambda}\left[G_{0 \bullet}\right]}{G_{0 p}}\right)^{2}}\right)
$$

For positive coupling constants $\lambda>0$ the angle function $\tau_{b}(a)$ ranges from 0 to $\pi$ and therefore gives rise to a winding number which manifests in an ambiguity in the formulae for $G_{a b}$ and $G_{0 b}$. A perturbative solution of (3.2) reproduces the Feynman graph expansion. However, for any $\lambda>0$ one leaves the radius of convergence of the arctan series so that the perturbative expansion does not converge. A better strategy is to solve (3.2) by iteration (and exactly in $\lambda<0$ ). This iteration converges numerically, and according to Figure 1 we find evidence for a second-order phase transition at critical coupling constant $\lambda_{c} \approx-0.39$.

\section{Schwinger functions and reflection positivity}

By reverting the matrix representation we convert the matrix correlation functions $G_{|\ldots|}$ to Schwinger functions in position space. Under conditions identified by Osterwalder-Schrader [8], the Fourier-Laplace transform of Schwinger functions gives rise to Wightman functions of a relativistic quantum field theory [1]. These conditions are [OSO] growth conditions, [OS1] Euclidean invariance, [OS2] reflection positivity, and [OS3] permutation symmetry. An additional axiom [OS4] clustering would give a unique vacuum state. 


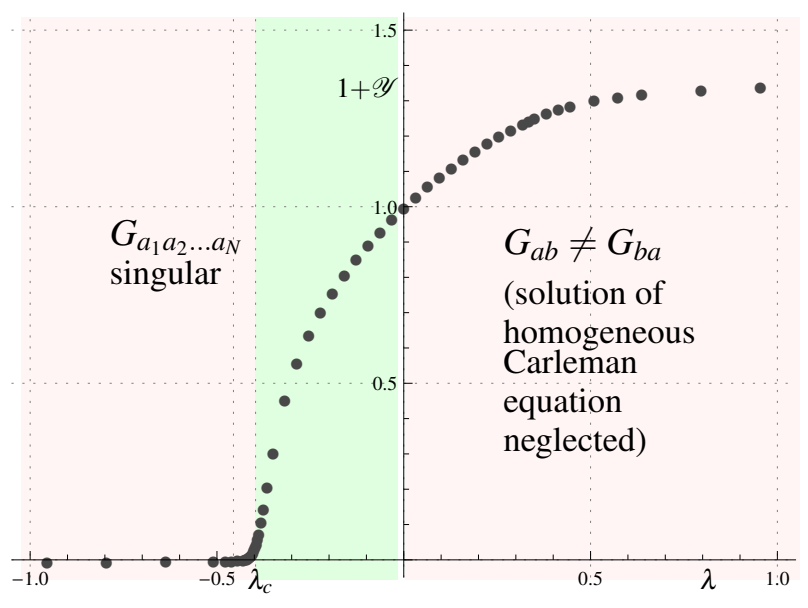

Figure 1: $1+\mathscr{Y}:=-\left.\frac{d G_{0 b}}{d b}\right|_{b=0}$ as function of $\lambda$, based on $G_{0 b}$ for $\Lambda^{2}=10^{7}$ with 2000 sample points.

Since the initial action (1.1) badly violates [OS1], it was completely clear to us that our model has no chance to satisfy the Osterwalder-Schrader axioms. To our enormous surprise, the infinite volume limit $\Theta \rightarrow \infty$ restored full Euclidean invariance:

Theorem 4 ([4]) The connected $N$-point Schwinger functions of the $\lambda \phi_{4}^{4}$-model on extreme Moyal space $\theta \rightarrow \infty$ are given by

$$
\begin{aligned}
S_{c}\left(\mu x_{1}, \ldots, \mu x_{N}\right)=\frac{1}{64 \pi^{2}} & \sum_{\substack{N_{1}+\ldots+N_{B}=N \\
N_{\beta} \text { even }}} \sum_{\sigma \in \mathscr{S}_{N}}\left(\prod_{\beta=1}^{B} \frac{4^{N_{\beta}}}{N_{\beta}} \int_{\mathbb{R}^{4}} \frac{d p_{\beta}}{4 \pi^{2} \mu^{4}} e^{\left.\mathrm{i}\left\langle\frac{p_{\beta}}{\mu}, \Sigma_{i=1}^{N_{\beta}}(-1)^{i-1} \mu x_{\sigma\left(N_{1}+\ldots+N_{\beta-1}+i\right)}\right\rangle\right)}\right. \\
\times & \underbrace{G}_{N_{1}} \underbrace{\frac{\left\|p_{1}\right\|^{2}}{2 \mu^{2}(1+\mathscr{Y})}, \cdots, \frac{\left\|p_{1}\right\|^{2}}{2 \mu^{2}(1+\mathscr{Y})}}_{N_{B}} \mid \cdots \underbrace{\frac{\left\|p_{B}\right\|^{2}}{2 \mu^{2}(1+\mathscr{Y})}, \cdots, \frac{\left\|p_{B}\right\|^{2}}{2 \mu^{2}(1+\mathscr{Y})}}
\end{aligned}
$$

Permutation symmetry [OS3] is trivially realised, and growth estimates [OSO] can be deduced from the integral equation (3.2). Clustering [OS4] is violated.

Only a restricted sector of the underlying matrix model contributes to position space: All strands of the same boundary component carry the same matrix index. The most interesting sec-

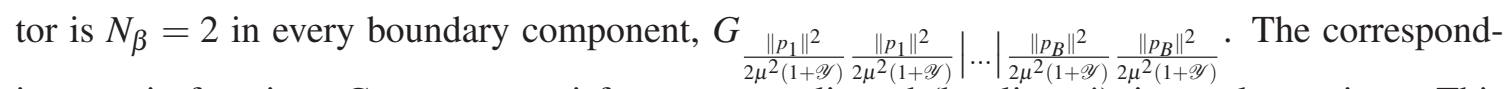
ing matrix functions $G_{a_{1} a_{1}|\ldots| a_{B} a_{B}}$ satisfy more complicated (but linear!) integral equations. This $(2+\ldots+2)$-sector describes the propagation and interaction of $B$ (Euclidean) particles without any momentum exchange. This is familiar from two-dimensional integrable models, but a sign of triviality in 4D. Typical triviality proofs rely on clustering or analyticity in Mandelstam representation. The validity of these assumptions in the present case needs verification.

Reflection positivity of $S_{c}\left(\mu x_{1}, \mu x_{2}\right)$ is equivalent [3] to the condition that $G_{a a}$ is a Stieltjes function, i.e. representable as $G_{a a}=\int_{0}^{\infty} \frac{d \rho\left(m^{2}\right)}{a+m^{2}}$ for a positive measure $\rho$. This representation, which can be checked by purely real conditions, defines a holomorphic continuation of $G_{a a}$ to the cut plane $\mathbb{C} \backslash[-\infty, 0]$ together with Minkowskian positivity $\operatorname{Im}\left(G_{a a}\right) \geq 0$ for $\operatorname{Im}(a)<0$. A discrete approximation as in Figure 1 cannot be holomorphic, but the Stieltjes property should fail in higher order for finer resolution. This is precisely what we observe (left of Figure 2). The improvement 

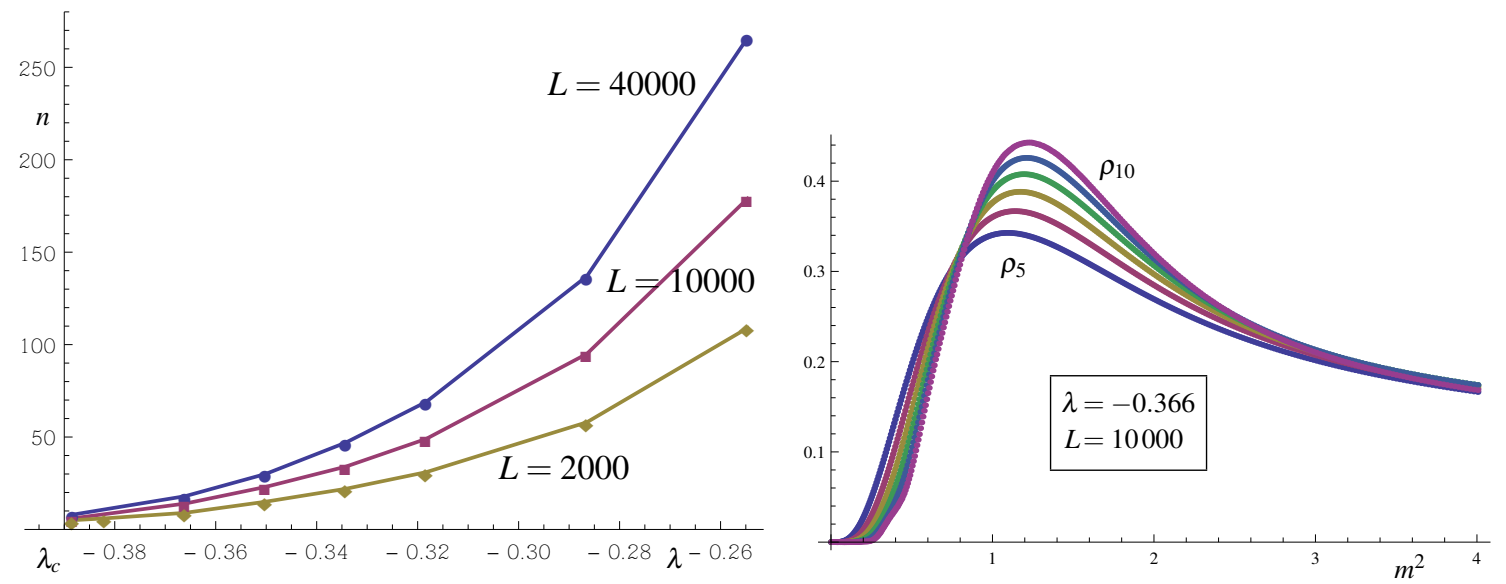

Figure 2: Left: Failure of logarithmically complete monotonicity $(-1)^{n}\left(\log G_{0 b}\right)^{(n)} \geq 0$ for various resolutions $L$ as function of $\lambda$. Right: The sequence $\rho_{k}$ of discrete approximations to the measure function $\rho\left(m^{2}\right)$ of $G_{a a}$.

slows down at precisely the same value $\lambda_{c} \approx-0.39$ as for the completely different problem of Figure 1. On the right of Figure 2 we show the first elements of a sequence $\rho_{k}$ which would converge to the measure $\rho$ if $G_{a a}$ is Stieltjes. Again we confirm positivity. Details are given in [4].

All this is clear evidence, albeit no proof, of reflection positivity of the Schwinger 2-point function $S_{c}\left(\mu x_{1}, \mu x_{2}\right)$ precisely in the phase $\lambda_{c}<\lambda \leq 0$.

\section{Acknowledgements}

R.W. would like to thank Thierry Masson for invitation to FFP14 and hospitality during the stay in Marseille.

\section{References}

[1] R. F. Streater and A. S. Wightman, PCT, spin and statistics, and all that, Benjamin, New York (1964).

[2] H. Grosse and R. Wulkenhaar, "Self-dual noncommutative $\phi^{4}$-theory in four dimensions is a non-perturbatively solvable and non-trivial quantum field theory," Commun. Math. Phys. 329 (2014) 1069-1130 [arXiv:1205.0465 [math-ph]].

[3] H. Grosse and R. Wulkenhaar, "Solvable limits of a 4D noncommutative QFT," arXiv:1306.2816 [math-ph].

[4] H. Grosse and R. Wulkenhaar, "Solvable 4D noncommutative QFT: phase transitions and quest for reflection positivity," arXiv:1406.7755 [hep-th].

[5] H. Grosse and R. Wulkenhaar, "Renormalisation of $\phi^{4}$-theory on noncommutative $\mathbb{R}^{4}$ in the matrix base," Commun. Math. Phys. 256 (2005) 305-374 [hep-th/0401128].

[6] M. Disertori, R. Gurau, J. Magnen and V. Rivasseau, "Vanishing of beta function of non commutative $\phi_{4}^{4}$ theory to all orders," Phys. Lett. B 649 (2007) 95-102 [hep-th/0612251].

[7] H. Grosse and R. Wulkenhaar, "Progress in solving a noncommutative quantum field theory in four dimensions," arXiv:0909.1389 [hep-th].

[8] K. Osterwalder and R. Schrader, "Axioms for Euclidean Green's functions II," Commun. Math. Phys. 42 (1975) 281-305. 